EPJ manuscript No.

(will be inserted by the editor)

\title{
Diffusion of gelation clusters in the Zimm model
}

\author{
Matthias Küntzel, Henning Löwe, Peter Müller, and Annette Zippelius \\ Institut für Theoretische Physik, Georg-August-Universität Göttingen, 37073 Göttingen, Germany
}

Version of October 29, 2018

\begin{abstract}
Starting from a Zimm model we study selfdiffusion in a solution of crosslinked monomers. We focus on the effects of the hydrodynamic interaction on the dynamics and the critical behaviour at the sol-gel-point. Hydrodynamic interactions cause the clusters' diffusion constant to depend not only on the cluster's size but also on the cluster's shape - in contrast to the Rouse model. This gives rise to a nontrivial scaling of the Kirkwood diffusion constant averaged over all clusters of fixed size $n, \widehat{D}_{n} \sim n^{-\widehat{b}}$ with $\widehat{b}=1 / d_{s}-1 / 2$ given in terms of the spectral dimension $d_{s}$ of critical percolation clusters. The long-time decay of the incoherent scattering function is determined by the diffusive motion of the largest clusters. This implies the critical vanishing $D_{\text {eff }} \sim \varepsilon^{a}$ of the cluster-averaged effective diffusion constant at the gel point with exponent $a=\left(3 / 2-\tau+1 / d_{s}\right) / \sigma$.
\end{abstract}

PACS. 64.60.Ht Dynamic critical phenomena-61.25.Hq Macromolecular and polymer solutions; polymer melts; swelling

\section{Introduction}

The relaxation of long lived density fluctuations in gelling polymer solutions is dominated by the translational diffusion of individual clusters 11. A broad distribution of cluster sizes and shapes gives rise to a corresponding distribution of relaxation times and a non-exponential decay of the incoherent scattering function $S(\boldsymbol{q}, t)$, which has been determined in several light scattering experiments 2 . 3.4.5. A stretched exponential is observed in the sol phase $S(\boldsymbol{q}, t) \sim \exp \left\{-\left(t / t_{q}\right)^{x}\right\}$, whereas at the critical point the decay is algebraic in time $S(\boldsymbol{q}, t) \sim t^{-y}$. The time scale of the stretched exponential diverges as the distance to the critical point $\varepsilon$ tends to zero, $t_{q} \sim \varepsilon^{-z}$. The mean relaxation time or inverse effective diffusion constant, de' fined by the time integral over the incoherent scattering function is observed to diverge with a different exponent, $D_{\text {eff }}^{-1} \sim \varepsilon^{-a}$. The experimentally determined exponents [2] 3.4. scatter considerably and are summarized in Table 1 together with theoretical predictions from Rouse dynamics 6.7 8 and Zimm dynamics (as derived below).

Table 1. Comparison of critical exponents for the incoherent scattering function.

\begin{tabular}{c|c|c|c|c|c} 
exponent & Zimm & Rouse & {$[2]$} & $\underline{3}$ & {$[4]$} \\
\hline$x$ & 0.80 & $1 / 2$ & 0.66 & $0.3-0.8$ & 0.64 \\
$y$ & 0.71 & 0.18 & 0.27 & $0.2-0.3$ & 0.34 \\
$z$ & 0.56 & 2.22 & 2.5 & - & - \\
$a$ & 0.16 & 1.82 & 1.9 & $0.5-1$ & 1.9
\end{tabular}

From a theoretical point of view the central question is: How does the cluster diffusion constant scale with molecular weight $n$ at the gel point? One expects $D_{n} \sim n^{-b}$ with an exponent $b$ that depends on the underlying dynamical model. Several dynamical models are commonly discussed in the literature [12,9. For Rouse dynamics $b=1$, independently of the topology of the clusters [6. With cluster statistics according to three-dimensional bond percolation, which is generally expected to be applicable, this gives rise 7,8 to $a=1.82$. The Zimm model takes into account hydrodynamic interactions, but has so far been solved only for linear chains 9. The Zimm prediction for linear chains is $b=1 / 2$, implying $D_{n} \sim 1 / R_{n}$ for phantom chains. It has been suggested [5] that this result should also hold for fractal structures, whose radius of gyration $R_{n}$ scales with the Hausdorff dimension $d_{f}$, giving rise to $D_{n} \sim n^{-1 / d_{f}}$. A different line of argument [2] starts from the Stokes-Einstein relation $D(R) \sim\left(k_{\mathrm{B}} T\right) /(\eta(R) R)$ to express the diffusion constant of a cluster of linear dimension $R$ in terms of its viscosity $\eta(R)$. Using $\eta(R) \sim R^{k / \nu}$, where $k$ is the critical exponent of the viscosity, one gets $D(R) \sim 1 / R^{1+k / \nu}$. Computer simulations [10 11] of dense melts obtain values of $b$ within the range of $0.69<b<$ 0.99 . We are not aware of estimates of $D_{n}$ from simulations that include solvent effects explicitly. However, in simulations of crosslinked particles, clusters of a given size $n$ coexist with a large number of smaller clusters which may give rise to hydrodynamic interactions in much the same way as solvent molecules [11.

It is our intention here to solve the preaveraged Zimm model for arbitrarily shaped clusters. We thereby determine the scaling behaviour $D_{n} \sim n^{-b}$ of cluster diffusion 
constants at the gel point and work out its implications for the long-time decay of $S(\boldsymbol{q}, t)$. A comparison to experimental values will be given in the Conclusions.

\section{Zimm dynamics}

\subsection{Hydrodynamic interactions}

We consider $N$ point-like monomers, which are characterized by their position vectors $\boldsymbol{R}_{i}, i=1, \ldots, N$, in threedimensional Euclidean space. Permanently formed crosslinks constrain $M$ randomly chosen pairs of particles $\left(i_{e}, j_{e}\right), e=1, \ldots, M$ and give rise to a distribution of molecular clusters of all shapes and sizes. Crosslinks are modelled by Hookean springs in the potential energy

$$
U:=\frac{3}{2 \ell^{2}} \sum_{e=1}^{M}\left(\boldsymbol{R}_{i_{e}}-\boldsymbol{R}_{j_{e}}\right)^{2}=: \frac{3}{2 \ell^{2}} \sum_{i, j=1}^{N} \boldsymbol{R}_{i} \cdot \Gamma_{i, j} \boldsymbol{R}_{j}
$$

The length $\ell>0$ plays the role of an inverse crosslink strength and physical units have been chosen such that $k_{\mathrm{B}} T=1$. A given crosslink configuration $\mathcal{G}=\left\{i_{e}, j_{e}\right\}_{e=1}^{M}$ is specified by its $N \times N$-connectivity matrix $\Gamma$ and can be decomposed uniquely into $K(\mathcal{G})$ disjoint clusters $\mathcal{N}_{k}$, which are the maximal subsets of monomers that are connected by crosslinks.

We study the dynamics of crosslinked monomers in the presence of a solvent fluid, giving rise to hydrodynamic interactions between the monomers. Purely relaxational dynamics in an incompressible fluid is described by the equation of motion 12,9

$$
\frac{\mathrm{d}}{\mathrm{d} t} \boldsymbol{R}_{i}(t)=-\sum_{j=1}^{N} \boldsymbol{H}_{i, j}\left(\boldsymbol{R}_{i}(t)-\boldsymbol{R}_{j}(t)\right) \frac{\partial U}{\partial \boldsymbol{R}_{j}(t)}+\boldsymbol{\eta}_{i}(t)
$$

with the mobility tensor

$$
\boldsymbol{H}_{i, j}(\boldsymbol{r}):=\delta_{i, j} \frac{1}{\zeta} \mathbf{1}+\left(1-\delta_{i, j}\right) \frac{1}{8 \pi \eta_{s} r}\left(\mathbf{1}+\hat{\boldsymbol{r}} \hat{\boldsymbol{r}}^{\dagger}\right) .
$$

The diagonal term in (3) accounts for a frictional force with friction constant $\zeta$ that acts when a monomer moves relative to the solvent. The non-diagonal term reflects the influence of the motion of monomer $j$ on the solvent at the position of monomer $i$ and is given by the Oseen tensor 13 14. Here $\eta_{s}$ denotes the solvent viscosity, $\delta_{i, j}$ the Kronecker symbol, $r:=|\boldsymbol{r}|, \hat{\boldsymbol{r}}:=\boldsymbol{r} / r, \mathbf{1}$ is the three-dimensional unit matrix and the dagger indicates the transposition of a vector. Rouse dynamics is recovered, if the non-diagonal terms of the mobility matrix are neglected. The Gaussian thermal-noise forces $\boldsymbol{\eta}_{i}(t)$ in (2) have zero mean and co-variance

$$
\overline{\boldsymbol{\eta}_{i}(t) \boldsymbol{\eta}_{j}^{\dagger}\left(t^{\prime}\right)}=2 \boldsymbol{H}_{i, j}\left(\boldsymbol{R}_{i}-\boldsymbol{R}_{j}\right) \delta\left(t-t^{\prime}\right) .
$$

Here $\delta$ stands for the Dirac-delta function and the overbar indicates the Gaussian average over all realizations of $\boldsymbol{\eta}$.

\subsection{Preaveraging approximation}

The equation of motion (2) is nonlinear due to the nonlinear dependence of the mobility on particles' positions. A simple but uncontrolled approximation is the so-called preaveraging approximation that was first introduced by Kirkwood and Risemann [14] and Zimm [15]. In this approximation the mobility matrix (3) is replaced by its expectation value $\left\langle\boldsymbol{H}_{i, j}\right\rangle_{\text {eq }}$, which is computed with the equilibrium distribution, i.e. the Boltzmann weight $\sim e^{-U}$. Due to rotational invariance of the potential (1) the averaged mobility matrix is a multiple of the identity matrix $\left\langle\boldsymbol{H}_{i, j}\left(\boldsymbol{R}_{i}-\boldsymbol{R}_{j}\right)\right\rangle_{\text {eq }}=\mathbf{1} \mathrm{H}_{i, j}^{\mathrm{eq}}$, where

$$
\mathrm{H}_{i, j}^{\mathrm{eq}}=\delta_{i, j} \frac{1}{\zeta}+\left(1-\delta_{i, j}\right) \frac{1}{6 \pi \eta_{s}}\left\langle\frac{1}{\left|\boldsymbol{R}_{i}-\boldsymbol{R}_{j}\right|}\right\rangle_{\mathrm{eq}} .
$$

In the computation of (5), care has to be taken of the zero eigenvalues of the connectivity matrix, corresponding to the translation of whole clusters. To this end we regularize the potential (1) by adding a confining term $\omega \sum_{i=1}^{N} \boldsymbol{R}_{i} \cdot \boldsymbol{R}_{i}$ and let $\omega>0$ tend to zero subsequently. The average in (5) is conveniently performed via the Fourier representation of $1 /|\boldsymbol{r}|$, and the result

$$
\begin{array}{r}
\left\langle\frac{1}{\left|\boldsymbol{R}_{i}-\boldsymbol{R}_{j}\right|}\right\rangle_{\mathrm{eq}}=\frac{1}{\ell} \sqrt{\frac{6}{\pi}} \lim _{\omega \downarrow 0}\left([G(\omega)]_{i, i}+[G(\omega)]_{j, j}\right. \\
\left.-2[G(\omega)]_{i, j}\right)^{-1 / 2}
\end{array}
$$

involves the resolvent $G(\omega):=(\Gamma+\omega)^{-1}$ of $\Gamma$. The limit $\omega \downarrow 0$ is taken by expanding the resolvent $G(\omega)=E_{0} / \omega+$ $Z+\mathcal{O}(\omega)$ in terms of $\omega$. Here $Z:=\left(1-E_{0}\right) / \Gamma$ is the pseudoinverse of the connectivity matrix and $E_{0}$ denotes the projector on the nullspace of $\Gamma$ which is spanned by the vectors which are constant when restricted to any one cluster. More precisely, the matrix element $\left[E_{0}\right]_{i, j}$ is given by the inverse number of monomers of the cluster if $i$ and $j$ are in the same cluster and zero otherwise (cf. section II.D. in [8] for details). Hence, the RHS of (6) vanishes for $\omega \downarrow 0$ whenever $i$ and $j$ belong to different clusters. Consequently, the preaveraged mobility matrix $\mathrm{H}^{\mathrm{eq}}$ shows correlations of different particles only if these particles are in the same cluster, in other words it is block-diagonal and within one block given by

$$
\mathrm{H}_{i, j}^{\mathrm{eq}}=\frac{1}{\zeta}\left[\delta_{i, j}+\left(1-\delta_{i, j}\right) h\left(\kappa^{2} \pi / \mathcal{R}_{i, j}\right)\right] .
$$

For convenience we introduced $\mathcal{R}_{i, j}:=Z_{i, i}+Z_{j, j}-2 Z_{i, j}$ and $h(x)=\sqrt{x / \pi}$. The parameter $\kappa:=\sqrt{6 / \pi} \zeta /\left(6 \pi \eta_{s} \ell\right)$ plays the role of the coupling constant of the hydrodynamic interaction. Formally setting $\kappa=0$ in (7) yields $\mathrm{H}_{i, j}^{\mathrm{eq}}=\zeta^{-1} \delta_{i, j}$, and the Zimm model for gelation reduces to the Rouse model for gelation $6,7,8$.

It is well known that the Oseen tensor does not give rise to a positive-definite mobility matrix for all possible spatial configurations of monomers. This defect is cured 
if the Rotne-Prager-Yamakawa tensor [16,17] is used instead. Again, the preaveraging procedure is done with a confining potential which is switched off afterwards. The function $h$ is then given by

$$
h(x)=\operatorname{erf}(\sqrt{x})-\frac{1}{\sqrt{\pi}} \frac{1-\mathrm{e}^{-x}}{\sqrt{x}} .
$$

It involves the error function [18] and reduces to $\sqrt{x / \pi}$ as $x \downarrow 0$.

As a result of preaveraging we obtain the Zimm model for crosslinked polymers in solution

$$
\frac{\mathrm{d}}{\mathrm{d} t} \boldsymbol{R}_{i}(t)=-\sum_{j=1}^{N} \mathrm{H}_{i, j}^{\mathrm{eq}} \frac{\partial U}{\partial \boldsymbol{R}_{j}(t)}+\boldsymbol{\eta}_{j}(t)
$$

with the co-variance of the thermal noise given by

$$
\overline{\boldsymbol{\eta}_{i}(t) \boldsymbol{\eta}_{j}^{\dagger}\left(t^{\prime}\right)}=2 \mathrm{H}_{i, j}^{\mathrm{eq}} \delta\left(t-t^{\prime}\right) \mathbf{1} \text {. }
$$

Since the connectivity matrix as well as $\mathrm{H}^{\mathrm{eq}}$ are blockdiagonal, it follows that clusters move independently of each other.

The Zimm equation (9) is linear, hence it can be solved exactly. This is most conveniently done by introducing new coordinates $\widetilde{\boldsymbol{R}}_{i}(t)$ through the coordinate transformation

$$
\boldsymbol{R}_{i}(t)=: \sum_{j=1}^{N}\left[\left(\mathrm{H}^{\mathrm{eq}}\right)^{1 / 2}\right]_{i, j} \widetilde{\boldsymbol{R}}_{j}(t)
$$

The resulting equation of motion for $\widetilde{\boldsymbol{R}}_{i}(t)$ coincides with the one for a monomer in the Rouse model 6 6 7 for crosslinked monomers, but with a formal connectivity ma$\operatorname{trix} \widetilde{\Gamma}:=\left(\mathrm{H}^{\mathrm{eq}}\right)^{1 / 2} \Gamma\left(\mathrm{H}^{\mathrm{eq}}\right)^{1 / 2}$. Its solution for (transformed) initial data $\widetilde{\boldsymbol{R}}_{i}\left(t_{0}\right)$ is given by (cf. Section II.C. in [8])

$$
\widetilde{\boldsymbol{R}}_{i}(t)=\sum_{j=1}^{N} \widetilde{U}_{i, j}\left(t-t_{0}\right) \widetilde{\boldsymbol{R}}_{j}\left(t_{0}\right)+\int_{t_{0}}^{t} \mathrm{~d} t^{\prime} \widetilde{U}_{i, j}\left(t-t^{\prime}\right) \widetilde{\boldsymbol{\eta}}_{j}\left(t^{\prime}\right)
$$

with uncorrelated random forces

$$
\overline{\widetilde{\boldsymbol{\eta}}_{i}(t) \widetilde{\boldsymbol{\eta}}_{j}^{\dagger}\left(t^{\prime}\right)}=2 \delta_{i, j} \delta\left(t-t^{\prime}\right) \mathbf{1}
$$

and the time evolution matrix $\widetilde{U}(t):=\exp \left(-3 t \widetilde{\Gamma} / \ell^{2}\right)$. The solution of the Zimm equation (9) is then obtained by inserting (12) in (11). For a discussion of (9) in the case of dendrimers see [19].

\subsection{Disorder average}

To complete the description of the dynamic model we have to specify the statistical ensemble that determines the realizations of crosslinks in the macroscopic limit $M \rightarrow \infty$, $N \rightarrow \infty$ with fixed crosslink concentration $c=M / N$. Two distributions of crosslinks will be considered. (i) Each pair of monomers is chosen independently with equal probability $c / N$, corresponding to mean-field percolation or random graphs. For $c<c_{\text {crit }}=1 / 2$ there is no macroscopic cluster and almost all clusters are trees 20]. Furthermore all trees of size $n$ are equally likely. (ii) Clusters are generated according to three-dimensional continuum percolation, which is closely related to the intuitive picture of gelation, where monomers are more likely to be crosslinked when they are close to each other. Since continuum percolation and lattice percolation are believed to be in the same universality class 21, we employ the scaling description of the latter 21, giving rise to a cluster-size distribution

$$
\tau_{n} \sim n^{-\tau} \exp \left\{-n / n^{*}\right\}
$$

for $\varepsilon \ll 1$ and $n \rightarrow \infty$ with a typical cluster size $n^{*}(\varepsilon) \sim$ $\varepsilon^{-1 / \sigma}$ that diverges as $\varepsilon \rightarrow 0$.

We denote by $\langle A\rangle$ the average of an observable $A(\mathcal{G})$ over all crosslink realizations $\mathcal{G}$. Partial averages

$$
\langle A\rangle_{n}:=\tau_{n}^{-1}\left\langle N^{-1} \sum_{k=1}^{K} \delta_{N_{k}, n} A\left(\mathcal{N}_{k}\right)\right\rangle
$$

of $A$ over all clusters with $n$ sites will be of particular interest. The normalization $\tau_{n}:=\left\langle N^{-1} \sum_{k=1}^{K} \delta_{N_{k}, n}\right\rangle$ represents the average number of clusters with $n$ sites per monomer. It is also known as the cluster-size distribution. By reordering the clusters, one gets the useful identity

$$
\sum_{n=1}^{\infty} n \tau_{n}\langle A\rangle_{n}=\left\langle\sum_{k=1}^{K}\left(N_{k} / N\right) A\left(\mathcal{N}_{k}\right)\right\rangle
$$

which is valid in the absence of an infinite cluster.

\section{Diffusion constants}

The diffusion constant of a cluster $\mathcal{N}_{k}$ with $N_{k}$ sites is commonly defined in terms of the long-time growth of the mean-squared displacement of its centre of mass $\boldsymbol{R}_{\mathrm{CM}}(t):=$ $N_{k}^{-1} \sum_{i \in \mathcal{N}_{k}} \boldsymbol{R}_{i}(t)$ according to

$$
\begin{aligned}
D\left(\mathcal{N}_{k}\right): & =\lim _{t \rightarrow \infty} \frac{1}{6 t} \overline{\left[\boldsymbol{R}_{\mathrm{CM}}(t)-\boldsymbol{R}_{\mathrm{CM}}(0)\right]^{2}} \\
& =\left(\sum_{i, j \in \mathcal{N}_{k}}\left[\frac{1}{\mathrm{H}^{\mathrm{eq}}}\right]_{i, j}\right)^{-1} .
\end{aligned}
$$

The expression (17) for the diffusion constant has already been derived in 22]. Another diffusion constant has been introduced by Kirkwood 9, 12

$$
\widehat{D}\left(\mathcal{N}_{k}\right):=\frac{1}{N_{k}^{2}} \sum_{i, j \in \mathcal{N}_{k}} \mathrm{H}_{i, j}^{\mathrm{eq}}
$$

It provides an upper bound to the former,

$$
D\left(\mathcal{N}_{k}\right) \leq \widehat{D}\left(\mathcal{N}_{k}\right)
$$


as can be shown by applying the Jensen-Peierls inequality to (17).

The diffusion constants (17) also determine the longtime behaviour of density fluctuations as described by the incoherent scattering function

$$
\begin{aligned}
S(\boldsymbol{q}, t \mid \mathcal{G}) & :=\lim _{t_{0} \rightarrow-\infty} \overline{\frac{1}{N} \sum_{i=1}^{N} \mathrm{e}^{\mathrm{i} \boldsymbol{q} \cdot\left[\boldsymbol{R}_{i}\left(t+t_{0}\right)-\boldsymbol{R}_{i}\left(t_{0}\right)\right]}} \\
& \stackrel{t \rightarrow \infty}{\sim} \sum_{k=1}^{K} \frac{N_{k}}{N} \exp \left\{-q^{2} t D\left(\mathcal{N}_{k}\right)\right\}
\end{aligned}
$$

in the stationary state $t_{0} \rightarrow-\infty$. Due to the blockdiagonal structure of $\Gamma$ and $\mathrm{H}^{\mathrm{eq}}$, contributions from individual clusters simply add up in (20).

\section{Results}

\subsection{Analytical results for the Kirkwood diffusion constant}

The scaling behaviour of the cluster-averaged Kirkwood diffusion constant at the critical point

$$
\widehat{D}_{n}:=\left.\langle\widehat{D}\rangle_{n}\right|_{c=c_{\text {crit }}}
$$

can be obtained from known results on random resistor networks. Starting from Eq. (7) we identify $\mathcal{R}_{i, j}$ as the resistance measured between any connected pair of vertices $(i, j)$ in a random resistor network 23. It is obtained from the network of crosslinked monomers by identifying each Hookean spring with a resistor of unit magnitude. Note that this is an exact correspondence. We will infer the critical behaviour of $\widehat{D}_{n}$ from that of the $p$-th moment $\rho_{n}^{(p)}:=\left.n^{-2} \sum_{i \neq j}^{n}\left\langle\mathcal{R}_{i, j}^{p}\right\rangle_{n}\right|_{c=c_{\text {crit }}}$. In fact, we will only need the special case $\rho_{n}^{(-1 / 2)}$ for this purpose. Actually, moments $\rho_{n}^{(p)}$ with $p$ other than $-1 / 2$ generically occur in these types of network problems, see e.g. the case $p=1$ in $[8$. For this reason and since it does not require more efforts, we treat straightaway the case with general $p$. In order to compute $\rho_{n}^{(p)}$, we consider

$$
\begin{aligned}
\sum_{n=2}^{\infty} n^{2} \tau_{n} \rho_{n}^{(p)} & =\int_{\mathbb{R}^{3}} \mathrm{~d}^{3} x \int_{0}^{\infty} \mathrm{d} R R^{p} P(R, \boldsymbol{x}) \\
& \sim \varepsilon^{-p \phi_{1}+(\tau-3) / \sigma} .
\end{aligned}
$$

Here $P(R, \boldsymbol{x}) \mathrm{d} R$ is the joint probability that two vertices of the random resistor network, whose relative position vector in $\mathbb{R}^{3}$ is $\boldsymbol{x}$, belong to the same cluster and that the resistance measured between them lies in the interval from $R$ to $R+\mathrm{d} R$ [24,25]. In fact, (22) coincides with Eq. (2.45) in 24. Its asymptotic behaviour as $\varepsilon \downarrow 0$ involves the first crossover exponent $\phi_{1}$ of random resistor networks, which determines how typical resistances scale with distance, $R \sim|\boldsymbol{x}|^{\phi_{1} / \nu}$. The validity of (22) requires that there is a divergence for $\varepsilon \downarrow 0$, i.e. that $p \phi_{1}-(\tau-3) / \sigma>0$.
This is particularly the case for $p=-1 / 2$ and threedimensional (and also mean-field) percolation exponents. Making a power-law ansatz for the $n$-dependence of $\rho_{n}^{(p)}$, we deduce from (22) and Eq. (3.9) in [8] that $\rho_{n}^{(p)} \sim n^{2 p \widehat{b}}$, where

$$
\widehat{b}:=\sigma \phi_{1} / 2=1 / d_{s}-1 / 2
$$

is expressed in terms of the spectral dimension $d_{s} 26$ 27. of critical percolation clusters. Hence, when using the preaveraged Oseen tensor in (77), i.e. $h(x)=x^{1 / 2}$, for the computation of the Kirkwood diffusion constant (18), we get $\zeta \widehat{D}_{n}=n^{-1}+\kappa \lambda n^{-\widehat{b}}$ with some constant $\lambda>0$. Thus $\widehat{D}_{n}$ shows a crossover from Rouse behaviour $\widehat{D}_{n} \sim n^{-1}$ for $n<\widehat{n}(\kappa) \sim \kappa^{-1 /(1-\widehat{b})}$ to Zimm behaviour

$$
\widehat{D}_{n} \sim n^{-\widehat{b}}
$$

for asymptotically large $n>\widehat{n}(\kappa)$. Since the radius of gyration of Gaussian phantom clusters with $n$ vertices scales as $R_{n} \sim n^{1 / d_{f}^{(\mathrm{G})}}$, where $d_{f}^{(\mathrm{G})}=1 / \widehat{b}$ is the associated fractal Hausdorff dimension 28, 29,30, Eq. (24) implies $\widehat{D}_{n} \sim 1 / R_{n}$ in the final asymptotic regime for $n$, as suggested in $[$.

Next we take a closer look at the Kirkwood diffusion constant $\widehat{D}_{n}$ for the special case of mean-field percolation. Clearly, by inserting the appropriate mean-field value $d_{s}=4 / 3$ [26,27] in [23), one immediately arrives at $\widehat{b}=1 / 4$. Alternatively, one can use the analytically known distribution [31] of distances on random trees to compute even the full asymptotic behaviour of $\left\langle\mathcal{R}_{i, j}^{p}\right\rangle_{n}$ for $p>-2$ as $n \rightarrow \infty$. To do so, we exploit that for mean-field percolation the average $\langle\bullet\rangle_{n}$ is over all $n^{n-2}$ equally weighted labelled tree clusters with $n$ vertices, see e.g. [8]. Hence, this average is in fact independent of the crosslink concentration $c$, and the resistance $\mathcal{R}_{i, j}$ reduces to the chemical distance between the vertices $i$ and $j$. This gives

$$
\begin{aligned}
\left\langle\mathcal{R}_{i, j}^{p}\right\rangle_{n} & =(n-2) ! \sum_{\rho=1}^{n-1} \frac{\rho^{p}(\rho+1)}{n^{\rho}(n-\rho-1) !} \\
& \stackrel{n \rightarrow \infty}{\sim} n^{p+1} \int_{0}^{1} \mathrm{~d} x x^{p+1} \exp \left[n \int_{0}^{x} \mathrm{~d} y \ln (1-y)\right],
\end{aligned}
$$

which is independent of $1 \leq i \neq j \leq n$. An asymptotic evaluation of the integral over $x$ for $n \rightarrow \infty$ by Laplace's method then yields

$$
\left\langle\mathcal{R}_{i, j}^{p}\right\rangle_{n} \stackrel{n \rightarrow \infty}{\sim} 2^{p / 2} \Gamma(1+p / 2) n^{p / 2},
$$

where $\Gamma$ denotes Euler's gamma function. By setting $p=$ $-1 / 2$, we arrive again at the mean-field exponent $\widehat{b}=1 / 4$ for $\widehat{D}_{n}$.

\subsection{Numerical results for the diffusion constant}

Now we turn to the averaged diffusion constant

$$
D_{n}:=\left.\langle D\rangle_{n}\right|_{c=c_{\text {crit }}}
$$




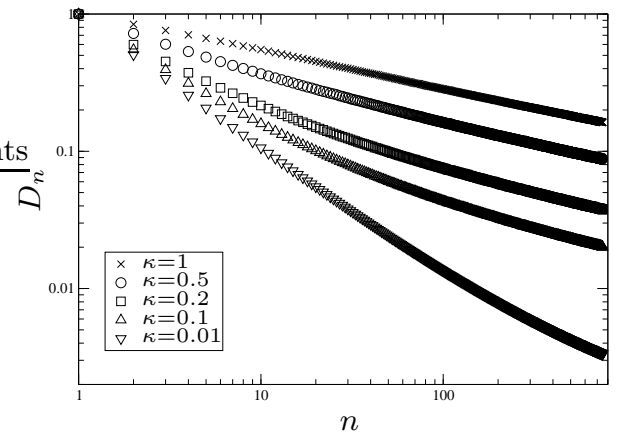

Fig. 1. $D_{n}$ at the gel point for mean field percolation and different hydrodynamic interaction strengths $\kappa$.

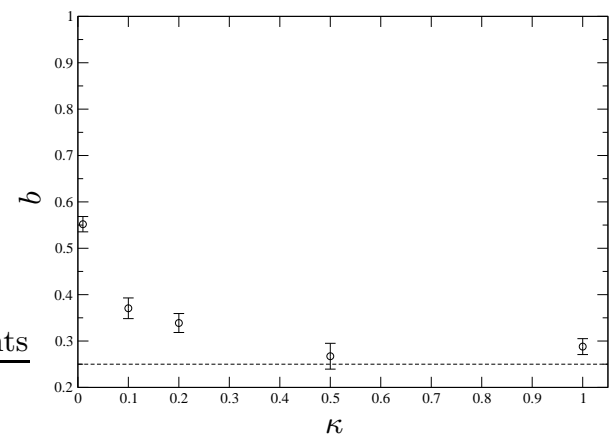

Fig. 2. Critical exponents $b$, corresponding to a power-law fit $D_{n} \sim n^{-b}$ in Fig. 1]

of clusters of size $n$ at the gel point. Assuming the critical scaling $D_{n} \sim n^{-b}$ for large cluster sizes $n$, the JensenPeierls inequality $D_{n} \leq \widehat{D}_{n}$, see (19), implies the inequality

$$
b \geq \widehat{b}
$$

for the critical exponents. More detailed information on $D_{n}$ is obtained from numerical studies: As already mentioned, for mean-field percolation the average $\langle\bullet\rangle_{n}$ is over all $n^{n-2}$ equally weighted labelled trees of size $n$ and hence independent of $c$. Labelled trees of a given size have been generated via the Prüfer-algorithm and handled with the LEDA-library 32. The preaveraged mobility matrix (7) is computed with $h$ corresponding to the Rotne-PragerYamakawa tensor. The resistances $\mathcal{R}_{i, j}$ in trees reduce to shortest graph-distances, which are computed with the Dijkstra algorithm 32 . For each $n=1 \ldots 750$ the diffusion constant (17) is averaged over 100 trees, which turned out to yield a reliable estimate for $D_{n}$. In Fig. 11 we plot $D_{n}$ as a function of $n$ on a double-logarithmic scale for different values of the hydrodynamic interaction parameter $\kappa$. The exponent $b$ is extracted by fitting the curves to a power law in the interval $n \in[700,750]$. Fig. 2] displays the exponent $b$ for different $\kappa$. The horizontal line marks the lower bound $\widehat{b}=1 / 4$ for $b$. A sharp crossover is observed from the Rouse value $b=1$ [6] for $\kappa=0$ to smaller values

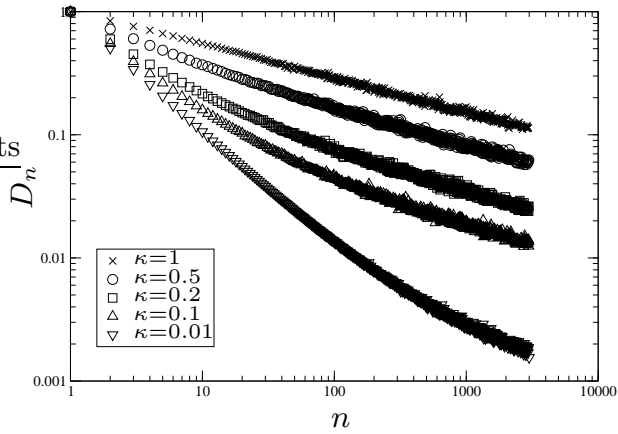

Fig. 3. $D_{n}$ at the gel point for three-dimensional bond percolation.

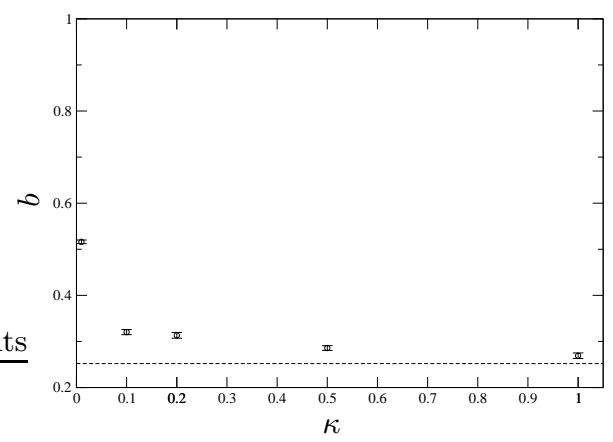

Fig. 4. Critical exponents $b$, corresponding to a power-law fit $D_{n} \sim n^{-b}$ in Fig. 3

of $b$ for non-zero $\kappa$. The latter are close to and may be identical to the lower bound $1 / 4$.

For the generation of clusters according to three-dimensional bond percolation we apply the Leath-Algorithm 33. It generates a sequence $\left\{\mathcal{N}_{l}\right\}_{l=1}^{L}$ of connected clusters, in terms of which the disorder average is readily computed via $\langle A\rangle=\lim _{L \rightarrow \infty} L^{-1} \sum_{l=1}^{L} A\left(\mathcal{N}_{l}\right)$. This implies $\langle A\rangle_{n}=\lim _{L \rightarrow \infty} \sum_{l=1}^{L} \delta_{N_{l}, n} A\left(\mathcal{N}_{l}\right) / \sum_{l=1}^{L} \delta_{N_{l}, n}$ for the average over clusters of size $n$. The algorithm has been tested by comparing the numerical data of the cluster-size distribution with the well-known scaling form of $\tau_{n}$. Additionally, for small values of $n$, we compare the number of clusters with known exact values 34. For each generated cluster the resistances $\mathcal{R}_{i, j}$ are computed from the MoorePenrose inverse $Z$ of the connectivity matrix $\Gamma$ - see below Eq. (7) - and inserted into (7) with $h$ corresponding to the Rotne-Prager-Yamakawa tensor. Due to high requirements of memory for the computation of the inverse of $\mathrm{H}^{\text {eq }}$ we restrict ourselves to cluster sizes $n<3000$. For small cluster sizes we compute up to 100 diffusion constants for given $n$, for large cluster sizes this number is considerably smaller. The total number of generated clusters lies in the range of 2151 for $\kappa=1$ and 5567 for $\kappa=0.01$. The results are shown in Figs. 3 and 4 In Fig. $3 D_{n}$ is plotted as a function of $n$ on a double-logarithmic scale for different values of $\kappa$. The exponent $b$, extracted by fitting the curves in Fig. 3 to a power law in the interval $n \in[500,3000]$, 
is shown in Fig. 4 The horizontal line marks the lower bound $\widehat{b} \approx 0.25$ for $b$, based on the value $d_{s} \approx 1.33[26$, 27. Again one may conjecture that $b=\widehat{b}$. Like the Kirkwood diffusion constant, $D_{n}$ also exhibits a crossover from Rouse to Zimm behaviour at a cluster size comparable to $\widehat{n}(\kappa)$.

\subsection{Incoherent scattering function}

Finally, we turn to the crosslink average $S(\boldsymbol{q}, t):=$ $\langle S(\boldsymbol{q}, t \mid \mathcal{G})\rangle$ of the incoherent scattering function (20) in the sol phase. By reordering the contributions of clusters as in (16), its long-time asymptotics is seen to be bounded from below by

$$
S(\boldsymbol{q}, t) \geq \sum_{n=1}^{\infty} n \tau_{n} \mathrm{e}^{-D_{n} q^{2} t} \geq \sum_{n=1}^{\infty} n \tau_{n} \mathrm{e}^{-\widehat{D}_{n} q^{2} t}
$$

according to the Jensen inequality and $D_{n} \leq \widehat{D}_{n}$. Using (14), the sum over $n$ on the RHS of (29) can be evaluated asymptotically for $\varepsilon \ll 1$ in terms of an integral. Up to a multiplicative constant, this yields $\left(q^{2} t\right)^{-y} s\left(t / t_{q}\right)$, where we introduced the typical relaxation time $t_{q} \sim q^{-2} \varepsilon^{-z}$, the critical exponents $y:=(\tau-2) / \widehat{b}$ and $z:=\widehat{b} / \sigma$ and the scaling function

$$
s(\lambda):=\lambda^{y} \int_{0}^{\infty} \mathrm{d} \alpha \alpha^{1-\tau} \exp \left[-\left(\alpha+\lambda \alpha^{-\widehat{b}}\right)\right] .
$$

The scaling function $s(\lambda)$ is of order unity as $\lambda \rightarrow 0$ and decays like a stretched exponential $s(\lambda) \sim \lambda^{x y} \exp \left[-\widetilde{\gamma} \lambda^{x}\right]$ with exponent $x:=(1+\widehat{b})^{-1}$ and some constant $\widetilde{\gamma}>0$ as $\lambda \rightarrow \infty$. In addition, we have verified numerically that the first inequality in (29) does not alter the long-time behaviour for $\varepsilon \ll 1$. Together with $b=\widehat{b}$, as suggested by Figs. 2] and 4, this implies that the desired scaling form of $S(\boldsymbol{q}, t)$ for $t \rightarrow \infty$ and $\varepsilon \ll 1$ is given by

$$
S(\boldsymbol{q}, t) \sim\left(q^{2} t\right)^{-y} s\left(t / t_{q}\right)
$$

Customarily, one defines an effective diffusion constant $D_{\text {eff }}$ by

$$
D_{\mathrm{eff}}^{-1}:=\lim _{q \rightarrow 0} q^{2} \int_{0}^{\infty} \mathrm{d} t S(\boldsymbol{q}, t)=\left.\sum_{n=1}^{\infty} n \tau_{n}\langle 1 / D\rangle_{n}\right|_{c=c_{\mathrm{crit}}} .
$$

We conclude from the scaling form (31) of $S(\boldsymbol{q}, t)$, that $D_{\text {eff }}$ vanishes at the gel point according to $D_{\text {eff }} \sim \varepsilon^{a}$ provided $a:=(2-\tau+\widehat{b}) / \sigma>0$. Three-dimensional bond percolation leads to the value $a \approx 0.16$. If instead $\widehat{b}<\tau-2$, then $D_{\text {eff }}$ remains non-zero at the transition. Such an unphysical situation will occur, for example, if one chooses meanfield percolation for the crosslink average. Note that the average time as exemplified by $D_{\text {eff }}$ is not proportional to the time scale $t_{q}$ of the stretched exponential, as is sometimes assumed incorrectly.

\section{Conclusions}

We have computed the averaged diffusion constant $D_{n}$ of clusters of a given size $n$ for Zimm dynamics at the gelation transition. Our main result, as suggested by the data, is the scaling $D_{n} \sim n^{-\widehat{b}}$. Here the exponent $\widehat{b}$ is given by Eq. (23), and the average is with respect to percolation statistics. Recalling that the radius of gyration of phantom clusters scales like $R_{n} \sim n^{\widehat{b}}$, the above relation shows that $D_{n} \sim 1 / R_{n}$ does not only hold for linear chains [9] but, in an average sense, for all percolation clusters.

Our results pertain to $\theta$-conditions, in so far as excluded-volume interactions have been ignored. One experimental setup to realize percolation statistics is crosslinking of a melt close to the gel point. Subsequent dilution is required to observe properties of single clusters as, for example, $D_{n}$. If, instead, one were to crosslink a dilute solution, one might generate different cluster statistics and hence different critical exponents.

The above scaling of the diffusion constants determines the critical dynamics of density fluctuations at the gelation transition within the framework of the Zimm model. For three-dimensional bond percolation the numerical values of the critical exponents are summarized in Table 1 and compared to the predictions of the Rouse model as well as to experimental values. Even though the latter scatter considerably, it is clear that the Zimm model fails to account for the observed critical behaviour. The failure can be traced to the very slow decrease of the diffusion constant with cluster size, resulting in too weak a divergence of the time scale and too fast a decay of the incoherent scattering function.

There are at least three reasons for the discrepancy between the predictions of the Zimm model and experiments: (i) It has been suggested [2] that hydrodynamic interactions between monomers on a cluster are screened by smaller clusters in the reaction bath so that the Rouse rather than the Zimm model should apply. Our analysis supports this conclusion in so far as the exponents of the Rouse model are closer to the experimental values. (ii) Excluded-volume interactions cause a swelling of the clusters whose influence on the diffusion constants can be estimated from a simple scaling argument: Provided the relation $D_{n} \sim 1 / R_{n}$ still holds in the presence of excluded-volume interactions, the scaling $R_{n} \sim n^{1 / d_{f}}$ can be obtained from a standard Flory argument [35] with the swollen fractal dimension $d_{f}=d_{s}(d+2) /\left(d_{s}+2\right)$. Here $d=3$ is the space dimension, and from $d_{s} \approx 4 / 3$ one gets $1 / d_{f} \approx 1 / 2$, which is roughly twice as large as $\widehat{b}$. The resulting exponent for the vanishing of the effective diffusion constant $D_{\text {eff }}$ is $a \approx 0.71$ for the case of three-dimensional bond percolation, respectively $a=0$ for mean-field percolation. Yet, these values for $a$ still lie well below the experimental ones. This may be due to the neglect of excluded-volume interactions between different clusters in this simple argument. (iii) Preaveraging of the hydrodynamic interactions is an uncontrolled approximation, and it remains to be seen what a full treatment of hy- 
drodynamic interactions predicts for the critical dynamics of gelling solutions.

This work was supported by the DFG through SFB 602 and Grant No. Zi 209/6-1.

\section{References}

1. P.-G. de Gennes, J. Physique (Paris) Lett. 40, L-197 (1979)

2. J. E. Martin, J. Wilcoxon, J. Odinek, Phys. Rev. A 43, 858 (1991).

3. M. Adam, M. Delsanti, J. P. Munch, D. Durand, Phys. Rev. Lett. 61, 706 (1988).

4. J. Bauer, W. Burchard, J. Phys. II (France) 2, 1053 (1992).

5. V. Lesturgeon, T. Nicolai, D. Durand, Eur. Phys. J. B 9, 71 (1999).

6. K. Broderix, P. M. Goldbart, A. Zippelius, Phys. Rev. Lett. 79, 3688 (1997).

7. K. Broderix, H. Löwe, P. Müller, A. Zippelius, Europhys. Lett. 48, 421 (1999).

8. K. Broderix, H. Löwe, P. Müller, A. Zippelius, Phys. Rev. E 63, 011510 (2001).

9. M. Doi, S. F. Edwards, The Theory of Polymer Dynamics (Clarendon Press, Oxford, 1988).

10. E. Del Gado, L. de Arcangelis, A. Coniglio, Eur. Phys. J. E 2, 359 (2000).

11. S. N. Jespersen, Phys. Rev. E 66, 031502 (2002).

12. R. B. Bird, C. F. Curtiss, R. C. Armstrong, O. Hassager, Dynamics of Polymeric Liquids, vol. 2 (Wiley, New York, 1987).

13. C. W. Oseen, Ark. Mat. Astr. Fys. 6, No. 29, 1 (1910).

14. J. G. Kirkwood, J. Riseman, J. Chem. Phys. 16, 565 (1948).

15. B. H. Zimm, J. Chem. Phys. 24, 269 (1956).

16. J. Rotne, S. Prager, J. Chem. Phys. 50, 4831 (1969).

17. H. Yamakawa, J. Chem. Phys. 53, 436 (1970).

18. M. Abramowitz, I. A. Stegun, Handbook of Mathematical Functions with Formulas, Graphs, and Mathematical Tables, ninth printing (Dover Publications, New York, 1972).

19. C. von Ferber, A. Blumen, J. Chem. Phys. 116, 8616 (2002).

20. P. Erdős, A. Rényi, Magyar Tud. Akad. Mat. Kut. Int. Közl 5, 17 (1960); reprinted in: P. Erdös: the Art of Counting, edited by J. Spencer (MIT Press, Cambridge, MA, 1973), Chap. 14, Article 324.

21. D. Stauffer, A. Aharony, Introduction to Percolation Theory (Taylor and Francis, London, 1994).

22. H. C. Öttinger, J. Chem. Phys. 87, 3156 (1987).

23. D. J. Klein, M. Randic̀, J. Math. Chem 12, 81 (1993).

24. A. B. Harris, T. C. Lubensky, Phys. Rev. B 35, 6964 (1987).

25. O. Stenull, H. K. Janssen, K. Oerding, Phys. Rev. E 59, 4919 (1999).

26. T. Nakayama, K. Yakubo, R. L. Orbach, Rev. Mod. Phys. 66, 381 (1994).

27. A. Bunde, S. Havlin, in: Fractals and disordered systems edited by A. Bunde, S. Havlin (Springer, Berlin, 1996), p. 59,115

28. M. E. Cates, J. Physique (France) 46, 1059 (1985).

29. T. A. Vilgis, Physica A 153, 341 (1988).

30. J.-U. Sommer, A. Blumen, J. Phys. A 28, 6669 (1995).
31. A. Meir, J. W. Moon, J. Combinatorial Theory 8, 99 (1970).

32. K. Mehlhorn, S. Näher, LEDA - A Platform for Combinatorial and Geometrical Computing (Cambridge University Press, Cambridge, 1999).

33. P. L. Leath, Phys. Rev. B 14, 5046 (1976).

34. M. F. Sykes, D. S. Gaunt, M. Glen, J. Phys. A 14, 287 (1981).

35. T. A. Vilgis, Phys. Rev. A 36, 1506 (1987). 